\title{
ISOLATING RED GIANTS IN M31'S OUTER SPHEROID: THE METALLICITY GRADIENT
}

\author{
D. REITZEL AND P. GUHATHAKURTA \\ UCO/Lick Observatory, Univ. of California, Santa Cruz \\ AND
}

A. GOULD

Dept. of Astronomy, Ohio State Univ.

The aim of our project is to measure the metallicity gradient in the spheroid of the Andromeda galaxy (M31). Deep KPNO 4-m UBRI images of a $15^{\prime} \times 15^{\prime}$ field in the outer spheroid of M31 are being used to isolate a sample of red giant branch (RGB) stars. These stars are distinguished from the more numerous distant field galaxies on the basis of broadband colors and image morphology. The color technique uses $U B R I$ photometry for isolating faint stars while rejecting $97 \%$ of the background galaxies (Gould et al. 1992). This yields a sample of candidate RGB stars in M31's spheroid, located about $20 \mathrm{kpc}$ from its center (in projection). The shape of the M31 RGB will be compared to those of Galactic globular clusters spanning a wide range of (known) metallicities. The metallicity of the M31 outer spheroid will be derived by interpolation. Combined with the metallicity measurement at $8.6 \mathrm{kpc}$ in M31's spheroid (Pritchet \& van den Bergh 1988), our new measurement will permit determination of the metallicity gradient of M31. This quantity is important for understanding galactic evolution as it provides a means to distinguish between the dissipational collapse model (Larson 1974) and the accretion model (Searle \& Zinn 1978).

\section{References}

Gould, A., Guhathakurta, P., Richstone, D. and Flynn, C. (1992) Evidence for Dwarf Stars at D 100 kpc Near the Sextans Dwarf Spheroidal Galaxy, Ap.J., 388, 345-353.

Larson, R.B. (1974) Dynamical Models for the Formation and Evolution of Spherical Galaxies, MNRAS, 166, 585-616.

Pritchet, C.J. and van den Bergh, S. (1988) Stellar Populations in the Inner Halo of M31, Ap.J., 331, 135-144.

Searle, L. and Zinn, R. (1978) Composition of Halo Clusters and the Formation of the Galactic Halo, Ap.J., 225, 357-379. 